\title{
Middle Latency Responses to Optimized Chirps in Adult Cochlear Implant Users
}

DOI: $10.3766 /$ jaaa. 18014

\author{
Razieh Alemi*†t \\ Alexandre Lehmann*†‡
}

\begin{abstract}
Background: Cochlear implant $(\mathrm{Cl})$ outcomes can be assessed using objective measures that reflect the integrity of the auditory pathway. One such measure is the middle latency response (MLR), which can provide valuable information for clinicians.

Purpose: Traditional stimuli for evoking MLRs, that is, clicks or tone bursts, do not stimulate all parts of the cochlea simultaneously, whereas chirp stimuli compensate for the cochlear neural delay and, therefore, produce more synchronous responses from the different neural elements of the cochlea. The purpose of the present study was to determine whether chirp stimuli can elicit reliable MLRs in $\mathrm{Cl}$ users and whether those responses correlate with clinical outcomes and with deprivation-related factors.
\end{abstract}

Research Design: We presented 2,000 free-field optimized chirp stimuli to $\mathrm{Cl}$ and control participants while their electroencephalography (EEG) was being recorded.

Study Sample: Twenty-four adult $\mathrm{Cl}$ users and 24 matched normal-hearing $(\mathrm{NH})$ individuals (age range from 18 to 63 years) participated in this study.

Data Collections and Analysis: The EEG was recorded from 64 active electrodes placed on the scalp. EEG signals were processed using EEGLAB and ERPLAB toolboxes. We characterized the latencies and amplitudes of the different MLR components in both groups.

Results: Chirp stimuli reliably evoked qualitatively similar MLRs across all NH and Cl participants with a couple of differences observed between the $\mathrm{NH}$ and $\mathrm{Cl}$ group. Among the different MLR components, the $\mathrm{Na}$ latency was significantly shorter for the $\mathrm{Cl}$ group. A significant amplitude difference was also found between groups for the $\mathrm{Pa}-\mathrm{Nb}$ complex, with higher amplitudes observed in the $\mathrm{NH}$ group. Finally, there were no significant correlations between MLR latencies (or amplitudes) and clinical outcomes or deprivation-related measures.

Conclusions: Free-field-presented optimized chirp stimuli were shown to evoke measurable and reliable MLRs in Cl users. In this experiment, the MLR morphology in $\mathrm{Cl}$ users was similar to those observed in $\mathrm{NH}$ participants. Even though we did not replicate here a significant relationship between MLR and speech perception measures, we were able to successfully collect acoustically evoked MLRs, which could constitute an important supplemental measure to the standard behavioral tests presently being used in postoperative clinical evaluation settings.

Key Words: chirp, cochlear implant, middle latency responses

Abbreviations: AEPauditory evoked potential; $\mathrm{Cl}=$ cochlear implant; $\mathrm{EEG}=$ electroencephalography; $\mathrm{HL}=$ hearing loss; MANOVA = multivariate analysis of variance; $\mathrm{MLR}=$ middle latency response; $\mathrm{NH}=$ normal-hearing; $\mathrm{SD}=$ standard deviation

*Department of Otolaryngology, Faculty of Medicine, McGill University, Montreal, QC, Canada; †Centre for Research on Brain, Language and Music (CRBLM), Montreal, QC, Canada; łInternational Laboratory for Brain, Music and Sound Research (BRAMS), Montreal, QC, Canada

Corresponding author: Razieh Alemi, Department of Otolaryngology, Faculty of Medicine, McGill University, Montreal, QC, Canada H4A 3J1; Email: razieh.alemi@mail.mcgill.ca

This work was funded by an Incubator Award from the Centre for Research on Brain, Language and Music (CRBLM). 


\section{INTRODUCTION}

$\mathrm{C}$ ochlear implants (CIs) can restore the lost sense of hearing by electrically stimulating the cochlear nerve. CIs can, therefore, provide the essential auditory inputs to the brain and promote cortical plasticity within its language-related areas (Moore and Shannon, 2009). Furthermore, CIs have been shown to significantly increase the quality of life and speech comprehension abilities in both children and adults (Svirsky et al, 2000; Vermeire et al, 2005).

However, the clinical outcomes of CI users vary greatly. The improvement in speech perception abilities appears to depend on a variety of factors including the etiology and the duration of hearing loss, the age of implantation, and other factors specific to the CI device (Blamey et al, 2013; Gama and Lehmann, 2015). A growing body of research is using auditory evoked potentials (AEPs) to objectively measure CI outcomes because they reflect the integrity of the auditory pathway (Shallop, 1993). AEPs are generally divided into three major classifications based on their latency: auditory brainstem responses, middle latency responses (MLRs), and finally, late AEPs (Picton, 2010).

MLRs are characterized by a series of sequential peaks and troughs within a poststimulus latency of 10-60 msec and are composed of the $\mathrm{P} 0, \mathrm{Na}, \mathrm{Pa}, \mathrm{Nb}$, and $\mathrm{Pb}$ components (McGee and Kraus, 1996). There are some advantages related to measuring MLRs over shorter latency AEPs. First, it has been shown that earlier evoked responses are more prone to stimulus artifact contamination than MLRs (Firszt et al, 2002). This susceptibility to stimulus artifacts might be due to stimulus length; for instance, it is more prudent to not let the offset of the stimulus overlap with the evoked response epoch. However, there are controversies in the literature regarding what constitutes the ideal stimulus length. For instance, a recent late AEP study showed that a stimulus length longer than the expected evoked potential can reduce stimulus artifact and prevent waveform peak corruption (Presacco et al, 2017). Contrary to this, Purdy and Kelly (2016) drew a different conclusion in their MLR study, where they found more artifacts in the responses evoked by long tonal stimuli in comparison to those elicited by brief stimuli used by Firszt et al (2002).

MLRs also constitute a reliable measure of neural survival (Jyung et al, 1989), can demonstrate the presence of central auditory processing disorders (Musiek et al, 2010), and can reveal plastic changes following CI (Gordon et al, 2005; Purdy and Kelly, 2016). Finally, MLRs have been successfully measured using acoustic and electrical stimuli in individuals with normal hearing $(\mathrm{NH})$, severe to profound hearing loss, and also in CI users (Kileny and Kemink, 1987; Kileny et al, 1989).
Several studies have examined the relationship between MLRs and clinical outcomes after implantation in adult and pediatric CI users (Pelizzone et al, 1989; Groenen et al, 1997; Makhdoum et al, 1998; Firszt et al, 2002; Gordon et al, 2005; Kelly et al, 2005; Kurnaz et al, 2009; Purdy and Kelly, 2016). However, the evidence suggesting a significant relationship is modest at best. For instance, Groenen et al (1997) found a significant relationship between electrically evoked MLRs and speech perception abilities in adult CI users, whereas other studies found no significant correlation (Makhdoum et al, 1998; Kelly et al, 2005; Kurnaz et al, 2009; Purdy and Kelly, 2016).

Findings regarding the relationship between objective measurements and CI experience or hearing loss duration vary significantly across studies. In a study on adult CI users, Kurnaz et al (2009) found no significant correlation between CI experience and MLR amplitudes/latencies, whereas Kelly et al (2005) indicated a negative correlation between the duration of hearing loss and the $\mathrm{Na}$ amplitude measured at the $\mathrm{Cz}$ electrode. In the pediatric population, Gordon et al (2005) showed an adverse effect of hearing loss duration on electrically evoked MLR changes. The authors concluded that MLR latencies decrease with increasing CI experience. However, the authors also highlighted the impact of age on the presence of MLRs in children, where evoked responses were more robust in older children, even in those with longer durations of hearing loss (Gordon et al, 2005).

One potential explanation for these inconsistent results in MLR studies with CI users may lie in the choice of auditory stimulus used to elicit the MLRs. Generally, MLRs in most studies have been obtained by using click or tone burst stimuli (Atcherson and Moore, 2014). Nevertheless, there are controversies regarding the MLR amplitudes evoked by such stimuli and the ability to reliably detect waveform components elicited by them (Woods et al, 1995; Nelson et al, 1997). For instance, a stimulus such as a click with an abrupt onset and a very short duration stimulates the different portions of the cochlea at different time points, beginning at the basal end of the cochlea and ending at its apex. Hence, click stimuli evoke temporally asynchronous neural cochlear responses that are unequal and out of phase (Atcherson and Moore, 2014).

To maximize the reliability of MLR responses and to overcome the problems associated with these stimuli, recent studies have begun to favor a different stimulus, known as a chirp stimulus (Dau et al, 2000; Elberling and Don, 2010). Chirp stimuli are designed to compensate for the temporal dispersion in the cochlea related to the traveling wave delay because the higher frequency components are delayed relative to lower frequency components (Dau et al, 2000; Elberling and Don, 2010; Atcherson and Moore, 2014). As a result, chirp stimuli produce larger amplitudes and more robust waveforms. 
Whether these optimal stimuli for MLRs are appropriate for CI users is presently unknown. If this were the case, then having a more robust response evoked by a standardized stimulus could shed light on the current controversy regarding the alleged relationship between MLRs and clinical outcomes in CI users. To date, no prior research has assessed the feasibility of using chirp stimuli to record MLRs in CI users. Therefore, the purpose of the present study was twofold: (a) to examine whether chirp stimuli can elicit reliable MLRs in adult CI users, as compared with $\mathrm{NH}$ matched controls, and (b) to determine whether those responses correlate with clinical outcomes (e.g., speech recognition scores) and/or deprivation-related measures (e.g., duration of hearing loss $[\mathrm{HL}]$ and CI experience). We used a freefield optimized chirp acoustic stimulus paradigm. Both adult CI users and $\mathrm{NH}$ controls were recruited to participate in the study.

\section{MATERIALS AND METHODS}

\section{Participants}

Twenty-four CI users, with a mean age of $43.50 \pm$ 14.63 years ( 17 female), and 24 paired $\mathrm{NH}$ individuals who were age and gender matched (mean age of $43.25 \pm$ 14.94 years) participated in this study (Table 1 ). The age range was from 18 to 63 years for both groups. Participants in the control group were tested for hearing acuity using tonal audiometry.

CI users were recruited through the Raymond-Dewar Institute and the MAB-MacKay Rehabilitation Center, two centers offering rehabilitation programs for the hearing impaired. Participants with self-reported neurological or psychological disorders were excluded from the study sample. All participants provided written informed consent before participating in the study. The study was approved by the Research Ethics Board of the Centre for Research in Rehabilitation of Greater Montreal.

\section{Stimuli Characteristics and Delivery}

Two thousand optimized chirp stimuli, with a frequency range of $200-8500 \mathrm{~Hz}$ and a duration of 7.2 msec, were presented to each participant with a mean stimulus onset asynchrony of $115 \mathrm{msec}(90-140 \mathrm{msec}$, uniformly jittered presentation). These chirp stimuli were designed based on a linear cochlea model and approximated the optimized chirp developed by Dau et al (2000). The starting and ending amplitudes for these frequency-sweeping and broadband chirps were zero. The stimuli were calibrated at 70-dB SPL using a SoundPro sound level meter (model DL 1/3 Octave Datalogging RTA; Quest, Bensenville, IL) and a fast rate mode with an Aweighting frequency filter. The speech processor of CI participants was kept on the "on position" and the magnet was
Table 1. Demographic Data of Participants in the $\mathrm{Cl}$ Group

\begin{tabular}{|c|c|c|c|c|c|}
\hline Code & $\begin{array}{c}\text { Age } \\
\text { (Years) }\end{array}$ & $\begin{array}{l}\text { Hearing } \\
\text { Loss } \\
\text { Duration } \\
\text { (Years) }\end{array}$ & $\begin{array}{c}\mathrm{Cl} \\
\text { Experience } \\
\text { (Years) }\end{array}$ & Device & $\begin{array}{c}\text { Speech } \\
\text { Recognition } \\
\text { Scores (\%) }\end{array}$ \\
\hline S01 & 22 & 22 & 14 & Cochlear & 56 \\
\hline S02 & 22 & 30 & 18 & Cochlear & 62 \\
\hline S03 & 43 & 37 & 7 & Cochlear & 34 \\
\hline SO4 & 51 & 14 & 12 & Cochlear & 76 \\
\hline S05 & 19 & 12 & 10 & $\begin{array}{c}\text { Advanced } \\
\text { Bionics }\end{array}$ & 72 \\
\hline S06 & 29 & 27 & 7 & Cochlear & 34 \\
\hline S07 & 63 & 23 & 3 & Neurelec & 84 \\
\hline S08 & 23 & 23 & 21 & Cochlear & 33 \\
\hline So9 & 35 & 11 & 9 & MED-EL & 33 \\
\hline S10 & 49 & 7 & 6 & MED-EL & 52 \\
\hline S11 & 56 & 3 & 3 & MED-EL & 44 \\
\hline S12 & 33 & 33 & 17 & $\begin{array}{c}\text { Advanced } \\
\text { Bionics }\end{array}$ & 52 \\
\hline $\mathrm{S} 13$ & 53 & 20 & 7 & $\begin{array}{c}\text { Advanced } \\
\text { Bionics }\end{array}$ & 68 \\
\hline S14 & 43 & 13 & 5 & $\begin{array}{c}\text { Advanced } \\
\text { Bionics }\end{array}$ & 34 \\
\hline S15 & 65 & 34 & 2 & $\begin{array}{c}\text { Advanced } \\
\text { Bionics }\end{array}$ & 56 \\
\hline S16 & 47 & 14 & 9 & Cochlear & 56 \\
\hline S17 & 51 & 44 & 17 & $\begin{array}{c}\text { Advanced } \\
\text { Bionics }\end{array}$ & 56 \\
\hline S18 & 41 & 20 & 17 & $\begin{array}{c}\text { Advanced } \\
\text { Bionics }\end{array}$ & 58 \\
\hline S19 & 57 & 37 & 4 & Neurelec & 78 \\
\hline S21 & 20 & 19 & 1 & $\begin{array}{c}\text { Advanced } \\
\text { Bionics }\end{array}$ & 36 \\
\hline S22 & 58 & 10 & 3 & Cochlear & 90 \\
\hline S23 & 58 & 18 & 3 & Neurelec & 80 \\
\hline S24 & 54 & 12 & 7 & Cochlear & 14 \\
\hline S25 & 52 & 27 & 1 & Cochlear & 80 \\
\hline
\end{tabular}

in place. Stimuli were presented from a single free-field loudspeaker positioned at the ear level $1.2 \mathrm{~m}$ away from participants, facing the implanted side (the proportion of right/left side presentations was matched across groups). Even though the sound-attenuated electroencephalography (EEG) room was not very reverberant, we took this step to ensure that the acoustic signal reached the participant's ear in the most direct manner, similar to our previously validated approach used for free-field brainstem recording protocols (Gama et al, 2017).

\section{EEG Recording}

The EEG was recorded from 64 active $\mathrm{Ag}-\mathrm{AgCl}$ electrodes (easy cap) placed over the scalp according to the international 10-10 electrode placement system (ActiveTwo; Biosemi B.V., Amsterdam, Netherlands), whereas participants were sitting comfortably in a chair in a sound-attenuated and electromagnetically 
shielded room. Participants were instructed to remain awake and immobile to minimize muscle artifacts.

Horizontal and vertical eye movements were recorded from bipolar electrodes placed on the lateral canthi and from periocular electrodes placed on the superior and inferior orbits. The ActiveTwo system provides impedance transformation on the electrode, thus allowing low-noise recordings free of interference currents. There was no need to control electrode impedances because the common mode sense active electrode and the driven right-leg passive electrode loop maintain the average potential close to the voltage of the analog to digital converter reference in the $\mathrm{AD}$ box (www.biosemi.com). Direct-current offsets were kept near zero when placing the electrodes. Reference-free electrode signals were amplified using an ActiveTwo amplifier (BioSemi) and sampled at $8192 \mathrm{~Hz}$. The signal was recorded and stored for offline analysis using BioSemi ActiView software.

\section{MLR Analysis}

EEG processing was carried out using EEGLAB (Delorme and Makeig, 2004) and ERPLAB (LopezCalderon and Luck, 2014) toolboxes, running under MATLAB 2016b (The MathWorks, Inc., Natick, MA). The signal was referenced to the average mastoids, and then resampled at $2048 \mathrm{~Hz}$ for further processing. EEG recordings were filtered using the ERPLAB plugin, with a 15-200 Hz cutoff IIR Butterworth filter (24 dB/octave,
4 th order). Continuous EEG was segmented into epochs from -20 to $+90 \mathrm{msec}$ relative to stimulus onset. Epochs containing artifacts were removed based on both visual inspection and EEGLAB's automatic iterative rejection procedure, with a threshold of five standard deviations (SDs). The mean rejected epoch percentages for the CI and $\mathrm{NH}$ groups were $6.7 \pm 3.6$ and $10.3 \pm 4.4$, respectively. Components containing CI-related artifacts were rejected using independent component analysis ("runica" algorithm implemented in the EEGLAB).

The peak latency of the three positive MLR components $(\mathrm{P} 0, \mathrm{~Pa}$, and $\mathrm{Pb})$ and of the two negative components ( $\mathrm{Na}$ and $\mathrm{Nb}$ ) measured at $\mathrm{FCz}$ were marked for further analysis. MLR components were defined automatically using the ERPLAB measurement tool, based on the following time windows: $18-23 \mathrm{msec}$ poststimulus onset for $\mathrm{P} 0,21-26 \mathrm{msec}$ for $\mathrm{Na}, 25-29 \mathrm{msec}$ for $\mathrm{Pa}, 29-36$ msec for $\mathrm{Nb}$ component, and, finally, 49-65 msec for $\mathrm{Pb}$. Peak-to-peak amplitudes were calculated based on the difference between the trough and the following positive peaks for $\mathrm{Na}-\mathrm{Pa}, \mathrm{Pa}-\mathrm{Nb}$, and $\mathrm{Nb}-\mathrm{Pb}$ complexes.

\section{Speech Recognition Test}

A validated list of 50 phonetically balanced French words was used to test speech recognition (Picard, 1997). This test was selected based on its availability in International French and its ease of administration. An open set of prerecorded monosyllable words was presented
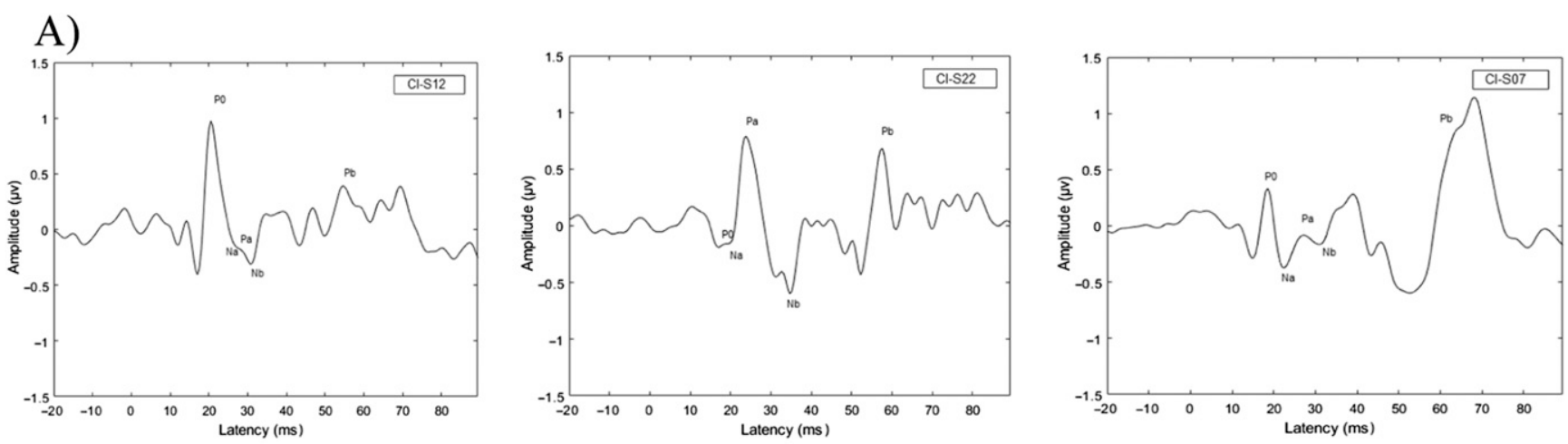

B)
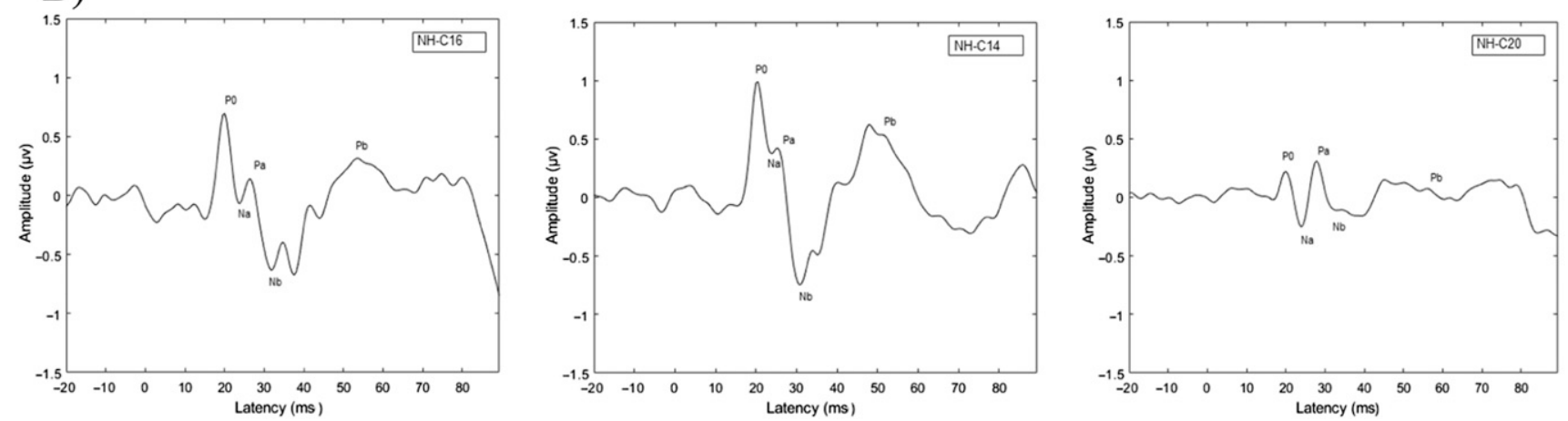

Figure 1. (A) MLR waveforms of three CI users (B) MLR waveforms of three NH participants. 
in the free field with a fixed presentation rate. After each word, participants were asked to repeat what they heard as quickly and as accurately as possible. No visual cues were provided during this part of the experiment. This behavioral test was performed during a separate session from the EEG recording, in the same sound-attenuated room, and presented at 70-dB SPL. The scores were calculated based on the percent of correctly repeated words. Only the CI users underwent the speech recognition test because we anticipated a ceiling effect in the $\mathrm{NH}$ group.

\section{Statistical Analysis}

Statistical analyses were performed using SPSS software (IBM Corp. 2015, V. 23.0; Armonk, NY). For quantitative measures, data are expressed as the mean \pm SD. MLR latencies and amplitudes were compared between the two groups using multivariate analysis of variance (MANOVA) analyses. The relationship between different component latencies/amplitudes and clinical outcomes/ deprivation-related variables was assessed using Pear- son correlations. For all statistical tests, the significance criterion was set at 0.05 , with Bonferroni adjustment of $p$-values for multiple testing.

\section{RESULTS}

hirp stimuli elicited typical and sizeable MLRs in $\mathcal{U}$ all $\mathrm{NH}$ and CI participants. Although there were some waveform morphology variations within participants, the morphology was found to be similar in MLR peaks and troughs across groups (Figure 1).

\section{Comparison of MLR Component Latencies between Groups}

Comparison of component latency values revealed a significant difference between groups $\left[F_{(5,42)}=2.93, p=\right.$ 0.023, Figure 2]. The MANOVA's between-subject effects (pairwise comparisons), using Bonferroni as an adjustment for multiple comparisons with an alpha level of $0.01(0.05 / 5$, as the number of comparisons for five

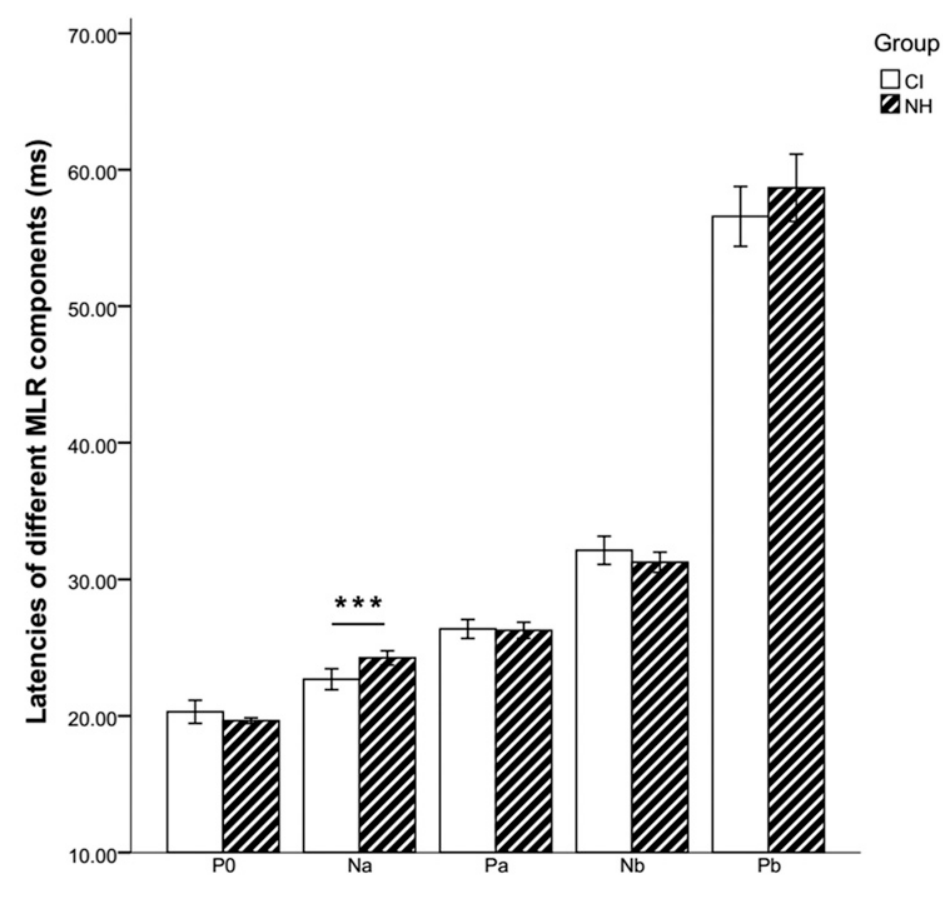

Latency: Mean $( \pm \mathrm{SD})$

\begin{tabular}{lccccc}
\hline Components/Groups & $\mathrm{P} 0$ & $\mathrm{Na}$ & $\mathrm{Pa}$ & $\mathrm{Nb}$ & $\mathrm{Pb}$ \\
\hline $\mathrm{CI}$ & $20.30 \pm 1.99$ & $22.68 \pm 1.80$ & $26.36 \pm 1.64$ & $32.12 \pm 2.43$ & $56.57 \pm 5.19$ \\
$\mathrm{NH}$ & $19.65 \pm 0.48$ & $24.25 \pm 1.23$ & $26.26 \pm 1.40$ & $31.25 \pm 1.75$ & $58.67 \pm 5.84$
\end{tabular}

Figure 2. Comparisons of peak latencies in different MLR components across individuals in CI and NH groups in FCz. Below the graph are the means $( \pm \mathrm{SD})$ of different MLR components across individuals in both groups. The error bar depicts the $95 \%$ confidence interval. $* * * p \leq 0.001$. 
components), revealed a significantly shorter Na component latency for CI users $(p=0.001)$. However, there were no other significant MLR latency differences (P0 component: $p=0.128$; Pa component: $p=0.819$; Nb component: $p=0.160$; $\mathrm{Pb}$ component: $p=0.196)$.

\section{Comparison of MLR Component Amplitudes between Groups}

Similar to what was observed for MLR latencies, the comparison of the $\mathrm{Na}-\mathrm{Pa}, \mathrm{Pa}-\mathrm{Nb}$, and $\mathrm{Nb}-\mathrm{Pb}$ complexes showed an overall significant group effect $\left[F_{(3,44)}=5.47, p=0.003\right.$, Figure 3]. Pairwise comparisons using a Bonferroni correction with an alpha value of $0.01(0.05 / 3$, as the number of comparisons for three complexes) showed a significant group difference for the $\mathrm{Pa}-\mathrm{Nb}$ complex $(p=0.004)$. The highest amplitude for the $\mathrm{Pa}-\mathrm{Nb}$ complex was observed in the $\mathrm{NH}$ group. Although the $\mathrm{NH}$ group showed higher amplitudes for the $\mathrm{Na}-\mathrm{Pa}$ and $\mathrm{Nb}-\mathrm{Pb}$ complexes, none of these differences were statistically significant $(\mathrm{Na}-\mathrm{Pa}$ complex: $p=0.38$, $\mathrm{Nb}-\mathrm{Pb}$ complex: $p=0.02$ ).

As a complementary step, another MANOVA was conducted on the individual component amplitudes. Comparison of individual component amplitude values revealed a significant group difference $\left[F_{(5,42)}=6.410\right.$, $p=0.000$, Figure 4]. Pairwise comparisons of betweensubject effects, with an alpha level of $0.01(0.05 / 5$, as the number of comparisons for five components), showed a significant group amplitude differences for $\mathrm{P0}(p=$ $0.000)$ and $\mathrm{Nb}(p=0.001)$. The highest amplitude values for $\mathrm{P0}$ and $\mathrm{Nb}$ components were observed in the $\mathrm{NH}$ group. CI group showed higher amplitude value for $\mathrm{Na}$ component, yet contrary to $\mathrm{Na}$ component's latency,

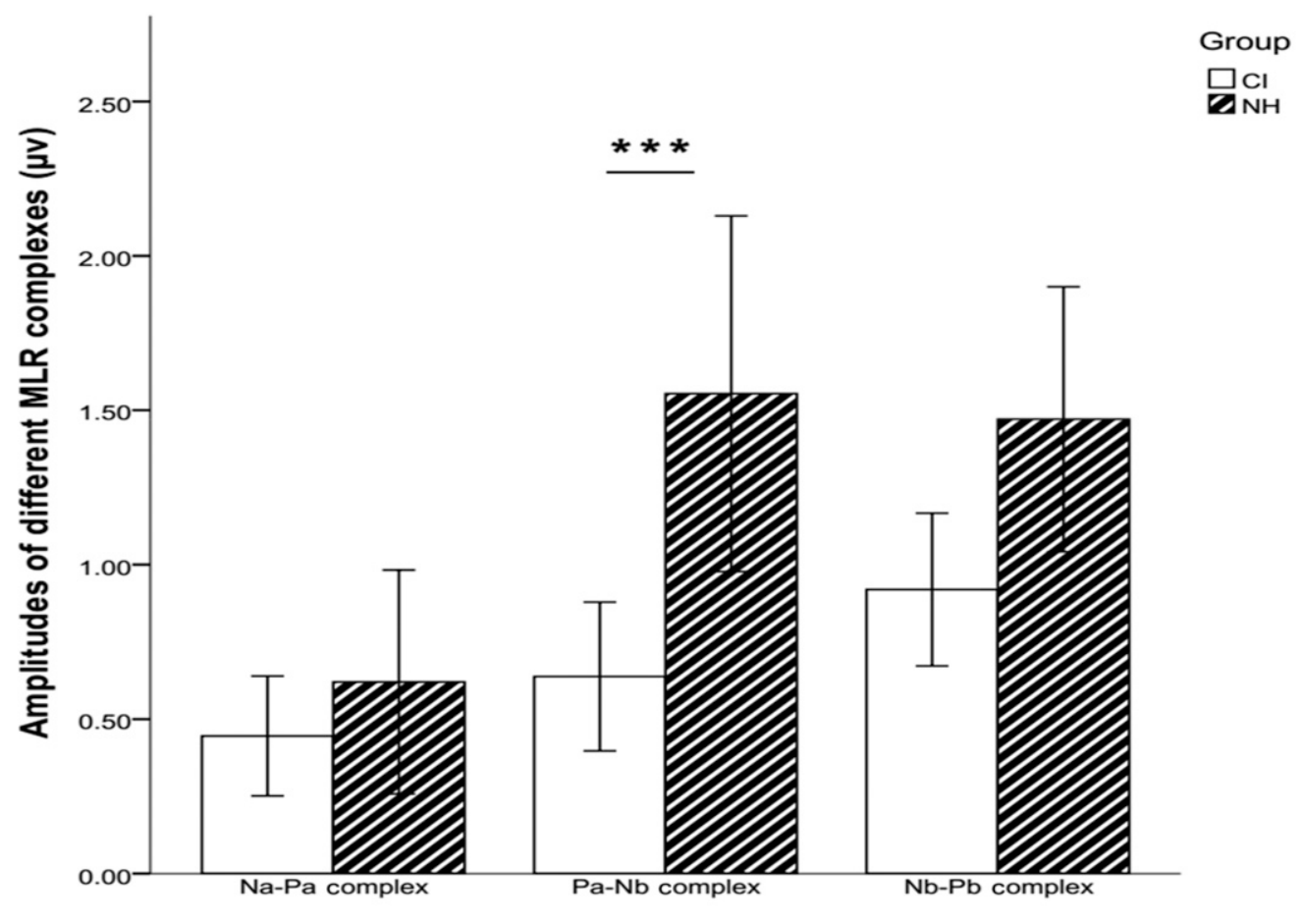

Amplitude: Mean $( \pm \mathrm{SD})$

Complexes/ Groups $\quad \mathrm{Na}-\mathrm{Pa} \quad \mathrm{Pa}-\mathrm{Nb} \quad \mathrm{Nb}-\mathrm{Pb}$

$\begin{array}{lrrr}\mathrm{CI} & .445 \pm .45 & .637 \pm .57 & .919 \pm .58 \\ \mathrm{NH} & .620 \pm .85 & 1.55 \pm 1.36 & 1.47 \pm 1.01\end{array}$

Figure 3. Comparisons of the amplitudes of different MLR complexes across individuals in CI and NH groups in FCz. Below the graph are the means $( \pm \mathrm{SD})$ of different MLR complexes across individuals in both groups. The error bar depicts the $95 \%$ confidence interval. $* * * p \leq 0.004$. 


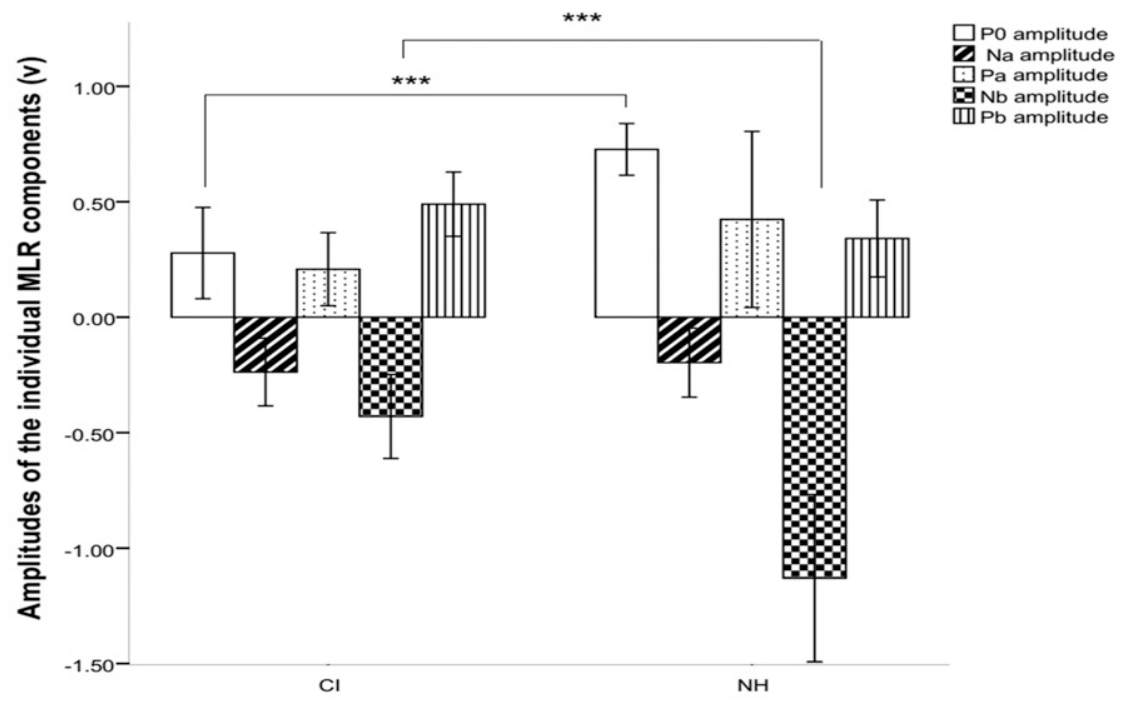

Amplitude: Mean $( \pm \mathrm{SD})$

\begin{tabular}{lccccc} 
Components/Groups & $\mathrm{P} 0$ & $\mathrm{Na}$ & $\mathrm{Pa}$ & $\mathrm{Nb}$ & $\mathrm{Pb}$ \\
\hline $\mathrm{CI}$ & $.277 \pm .46$ & $-.237 \pm .34$ & $.207 \pm .37$ & $-.430 \pm .43$ & $.489 \pm .33$ \\
$\mathrm{NH}$ & $.726 \pm .26$ & $-.196 \pm .35$ & $.423 \pm .90$ & $-1.12 \pm .85$ & $.340 \pm .39$
\end{tabular}

Figure 4. Comparisons of individual amplitudes of different MLR components in CI and NH groups in FCz. The error bar depicts the 95\% confidence interval. Below the graph are the means $( \pm \mathrm{SD})$ of different MLR components in both groups. $* * * p \leq 0.001$.

this difference was not significant ( $p=0.688$ ). No other significant group differences were found ( $\mathrm{Pa}$ component: $p=0.285$; Pb component: $p=0.164)$.

\section{Correlation between MLR Amplitudes/Latencies and Clinical Outcomes/Deprivation- Related Factors}

Pearson correlations were performed to evaluate the relationship between MLRs and clinical outcomes or deprivation-related factors. Results revealed that there were no significant correlations between the complex amplitudes and the speech recognition scores, the HL duration, or the time elapsed since implantation, all $p>0.05$ (Table 2). Similarly, there were no significant correlations between the different peak latencies and the speech recognition scores, the HL duration or the time since implantation, all $p>0.05$ (Table 3).

\section{DISCUSSION}

\section{General Statement}

The current experiment has indicated that it is possible to use optimized chirp stimuli delivered in the free field to elicit reliable MLRs in CI users. We also showed

Table 2. The Correlation between the Amplitude of MLR Complexes and Speech Recognition Scores, HL Duration, and Cl Experience in the $\mathrm{Cl}$ Group

\begin{tabular}{|c|c|c|c|c|c|c|}
\hline & \multicolumn{2}{|c|}{$\mathrm{Na}-\mathrm{Pa}$} & \multicolumn{2}{|c|}{$\mathrm{Pa}-\mathrm{Nb}$} & \multicolumn{2}{|c|}{$\mathrm{Nb}-\mathrm{Pb}$} \\
\hline & $r$ & $\overline{p \text {-Value }}$ & $R$ & $\overline{p \text {-Value }}$ & $r$ & $\overline{p \text {-Value }}$ \\
\hline Speech recognition scores & 0.299 & 0.213 & 0.122 & 0.619 & -0.049 & 0.841 \\
\hline $\mathrm{HL}$ duration & -0.178 & 0.417 & -0.319 & 0.138 & 0.037 & 0.869 \\
\hline $\mathrm{Cl}$ experience & 0.078 & 0.723 & 0.087 & 0.691 & -0.088 & 0.690 \\
\hline
\end{tabular}

$p>0.05$. 
Table 3. The Correlation between the Latencies of Different MLR Components and Speech Recognition Scores, HL Duration, and $\mathrm{Cl}$ Experience in the $\mathrm{Cl}$ Group

\begin{tabular}{|c|c|c|c|c|c|c|c|c|c|c|}
\hline & \multicolumn{2}{|c|}{$\mathrm{PO}$} & \multicolumn{2}{|c|}{$\mathrm{Na}$} & \multicolumn{2}{|c|}{$\mathrm{Pa}$} & \multicolumn{2}{|c|}{$\mathrm{Nb}$} & \multicolumn{2}{|c|}{$\mathrm{Pb}$} \\
\hline & $r$ & $p$-Value & $r$ & $p$-Value & $r$ & $p$-Value & $r$ & $p$-Value & $r$ & $p$-Value \\
\hline Speech recognition scores & 0.011 & 0.961 & -0.372 & 0.081 & 0.295 & 0.172 & 0.286 & 0.186 & -0.066 & 0.766 \\
\hline HL duration & -0.178 & 0.416 & 0.376 & 0.077 & -0.168 & 0.442 & -0.196 & 0.370 & 0.098 & 0.656 \\
\hline $\mathrm{Cl}$ experience & -0.013 & 0.954 & 0.386 & 0.069 & -0.266 & 0.221 & 0.280 & 0.196 & -0.395 & 0.062 \\
\hline
\end{tabular}

$p>0.05$

that the morphology of the MLR components evoked by chirp stimuli in adult CI users is similar to that measured in NH individuals (Figure 1). However, some individual MLR-based components significantly differed between CI users and NH controls. For instance, the $\mathrm{Na}$ component latency was significantly shorter in the CI group, whereas this difference was not significant for other components (Figure 2). Contrary to our current results, Kurnaz et al (2009) indicated a longer latency for the Na component in CI users. This discrepancy might be due to the significantly smaller number of participants in their study (6 versus 21 postlingual participants in the present study; Table 1). Interestingly, our CI group showed higher $\mathrm{Na}$ amplitudes than the NH group; nevertheless, this difference was not statistically significant (Figure 4). Conversely, we found a significant amplitude group difference for the $\mathrm{Pa}-\mathrm{Nb}$ complex, with larger amplitudes observed in the $\mathrm{NH}$ group (Figure 3).

Finally, in contrast to the findings of Groenen et al (1997), we did not observe any significant correlations between MLR latency/amplitude measures with speech recognition scores, and between MLR latency/amplitude measures and deprivation-related characteristics. These results are in-line with the bulk of literature (Makhdoum et al, 1998; Kelly et al, 2005; Kurnaz et al, 2009; Purdy and Kelly, 2016) (Tables 2 and 3).

\section{Chirp Stimuli as a Reliable Alternative to Click Stimuli for Eliciting MLRs}

Chirp stimuli have the advantage of being able to stimulate the different regions of the basilar membrane at the same time, thus increasing temporal synchrony and evoking larger response amplitudes (Elberling and Don, 2010; Atcherson and Moore, 2014). This stimulus feature has led to the widespread use of chirp stimuli for recording auditory brainstem responses and MLRs in NH participants (Dau et al, 2000; Atcherson and Moore, 2014). In the present study, we showed that it is also feasible to record MLRs in CI users elicited by chirp stimuli presented under free-field listening conditions. Because most studies have used electrical stimulation to record MLRs from CI users (Hoth and Dziemba, 2017), the present findings demonstrated the significant potential of the free-field presented chirp stimuli for the evaluation of CIs in clinical settings. However, more studies are needed to properly determine the clinical value of the measures obtained with the chirp stimulus.

\section{Evaluating the Relationship between MLRs and Speech Recognition Abilities}

Although it has been documented that experience with CI leads to a significant improvement in speech perception ability, especially during the first year of CI use (Blamey et al, 2013; Moberly et al, 2017), it does not imply that objective MLR measures are necessarily directly related to this ability. To our knowledge, only one study to date has shown a significant correlation between interelectrode amplitude variations (and latency measures) with speech perception scores (Groenen et al, 1997). By contrast, we did not find a significant correlation between MLR features and speech recognition abilities in the present study. Speech perception relies on an interactive process between linguistic, cognitive, and social information, where a vast neural network contributes to the analysis of the different aspects of the speech stimulus (Moberly et al, 2017; Alemi et al, 2018). Therefore, it is perhaps not surprising that we could not find any evidence linking speech perception abilities to a single electrophysiological metric that is likely driven by a minority of these processes.

\section{Study Limitations}

This study's main goals were to determine whether chirp stimuli can elicit reliable MLRs in CI users and to ascertain whether those responses correlate with clinical outcomes and with plasticity-related factors. One limitation of the present study was that we did not measure speech recognition scores or MLRs longitudinally.

Another limitation of the present study was related to CI processors strategies. Preprocessing strategies used in CI sound processors take advantage of statistical rules to promote overall sound clarity and increase speech intelligibility in the presence of background noise and challenging listening environment. These strategies apply a combination of signal processing and noise reduction algorithms, along with microphone gain compression and sensitivity control. Yet, there are 
challenges related to infinite compression for stimuli surpassing $65 \mathrm{~dB}$ SPL, that is, sound distortion for incoming stimuli (Rakszawski et al, 2016). In the current experiment, the stimuli were presented acoustically in free field at $70 \mathrm{~dB}$, so there was a possibility for sound compression on some devices. Despite this limitation, reliable neural responses were elicited in our study. In the future, additional studies should consider using a lower intensity stimulation to avoid compression. It would also be interesting to compare MLRs obtained via free-field stimulation with those obtained electrically at this cutoff stimulus intensity.

The third limitation of this study was related to using only one type of auditory stimuli for eliciting MLRs in CI users. A recent study that demonstrated the clinical advantages of using chirp for eliciting MLRs did so by comparing MLRs evoked by chirp with MLRs evoked by click and tone burst stimuli in $\mathrm{NH}$ participants (Atcherson and Moore, 2014). Confirming the superiority of chirp stimuli over other traditional stimuli for CI users may require the direct comparison of MLRs evoked by these different stimuli in the same participants.

\section{CONCLUSION}

$\mathrm{C}$ ompared with other stimuli typically used to elicit MLRs in NH listeners, chirp stimuli take into account the cochlear traveling wave delay and generate higher temporal synchrony within neural structures. In the present study, we demonstrated the feasibility of recording MLRs elicited by free-field chirp stimuli in CI users. Although we established that MLRs could be reliably recorded in CI users, we did not find evidence that they could be used as neural markers of speech recognition abilities. MLRs are critical for the postoperative monitoring of auditory pathway function, whereas individuals learn to adapt to the new auditory inputs. Consequently, acoustically evoked MLRs could constitute an essential supplement to the standard behavioral tests presently being used in postoperative clinical evaluation settings.

Acknowledgments. The recruitment of CI users for this study was made possible, thanks to the Raymond-Dewar Institute and the MAB-MacKay Rehabilitation Center. The authors would also like to thank Dr. Patrice Voss for his excellent comments on the manuscript and Marie-Anne Prudhomme, Frédérik Desaulniers, and Mihaela Felezeu for their help in recording EEG.

\section{REFERENCES}

Alemi R, Batouli SAH, Behzad E, Ebrahimpoor M, Oghabian MA. (2018) Not single brain areas but a network is involved in language: applications in presurgical planning. Clin Neurol Neurosurg 165:116-128.
Atcherson SR, Moore PC. (2014) Are chirps better than clicks and tonebursts for evoking middle latency responses? J Am Acad Audiol 25(6):576-583.

Blamey P, et al. (2013) Factors affecting auditory performance of postlinguistically deaf adults using cochlear implants: an update with 2251 patients. Audiol Neurootol 18(1):36-47.

Dau T, Wegner O, Mellert V, Kollmeier B. (2000) Auditory brainstem responses with optimized chirp signals compensating basilar-membrane dispersion. J Acoust Soc Am 107(3): 1530-1540.

Delorme A, Makeig S. (2004) EEGLAB: an open source toolbox for analysis of single-trial EEG dynamics including independent component analysis. J Neurosci Methods 134(1):9-21.

Elberling C, Don M. (2010) A direct approach for the design of chirp stimuli used for the recording of auditory brainstem responses. J Acoust Soc Am 128(5):2955-2964.

Firszt JB, Chambers RD, Kraus N, Reeder RM. (2002) Neurophysiology of cochlear implant users I: effects of stimulus current level and electrode site on the electrical ABR, MLR, and N1-P2 response. Ear Hear 23(6):502-515.

Gama N, Peretz I, Lehmann A. (2017) Recording the human brainstem frequency-following-response in the free-field. $J$ Neurosci Methods 280:47-53.

Gama NM, Lehmann A. (2015) Commentary: "compensatory plasticity: time matters" Front Neurosci 9:348.

Gordon KA, Papsin BC, Harrison RV. (2005) Effects of cochlear implant use on the electrically evoked middle latency response in children. Hear Res 204(1):78-89.

Groenen P, Snik A, van den Broek P. (1997) Electrically evoked auditory middle latency responses versus perception abilities in cochlear implant users. Audiology 36(2):83-97.

Hoth S, Dziemba OC. (2017) The role of auditory evoked potentials in the context of cochlear implant provision. Otol Neurotol 38(10): e522-e530.

Kelly AS, Purdy SC, Thorne PR. (2005) Electrophysiological and speech perception measures of auditory processing in experienced adult cochlear implant users. Clin Neurophysiol 116(6): $1235-1246$.

Kileny PR, Kemink JL. (1987) Electrically evoked middle-latency auditory potentials in cochlear implant candidates. Arch Otolaryngol Head Neck Surg 113(10):1072-1077.

Kileny PR, Kemink JL, Miller JM. (1989) An intrasubject comparison of electric and acoustic middle latency responses. Otol Neurotol 10(1):23-27.

Kurnaz M, Satar B, Yetiser S. (2009) Evaluation of cochlear implant users' performance using middle and late latency responses. Eur Arch Otorhinolaryngol 266(3):343-350.

Jyung RW, Miller JM, Cannon SC. (1989) Evaluation of eighth nerve integrity by the electrically evoked middle latency response. Otolaryngol Head Neck Surg 101(6):670-682.

Lopez-Calderon J, Luck SJ. (2014) ERPLAB: an open-source toolbox for the analysis of event-related potentials. Front Hum Neurosci 8:1-14.

Makhdoum MJ, Groenen PAP, Snik AFM, van den Broek P. (1998) Intra-and interindividual correlations between auditory evoked 
potentials and speech perception in cochlear implant users. Scand Audiol 27(1):13-20.

McGee T, Kraus N. (1996) Auditory development reflected by middle latency response. Ear Hear 17(5):419-429.

Moberly AC, Harris MS, Boyce L, Nittrouer S. (2017) Speech recognition in adults with cochlear implants: the effects of working memory, phonological sensitivity, and aging. J Speech Lang Hear Res 60(4):1046-1061.

Moore DR, Shannon RV. (2009) Beyond cochlear implants: awakening the deafened brain. Nat Neurosci 12(6):686-691.

Musiek FE, Baran JA, Bellis TJ, Chermak GD, Hall JW, Keith RW, Medwetsky L, West KL, Young M, Nagle S. (2010) Guidelines for the Diagnosis, Treatment and Management of Children and Adults with Central Auditory Processing Disorder. American Academy of Audiology https://audiology-web.s3.amazonaws.com/migrated/CAPD\%20Guidelines\%208-2010.pdf_539952af956c79.73897613.pdf. Accessed April 24, 2019.

Nelson MD, Hall JW, Jacobson GP. (1997) Factors affecting the recordability of auditory evoked response component $\mathrm{Pb}(\mathrm{P} 1) \mathrm{J}$ Am Acad Audiol 8(2):89-99.

Pelizzone M, Kasper A, Montandon P. (1989) Electrically evoked responses in cochlear implant patients. Audiology 28(4):230-238.

Picard M. (1997) Speech audiometry in French-speaking Quebec. J speech Lang Pathol Audiol 21(4):301-311.
Picton TW. (2010) Human Auditory Evoked Potentials. San Diego, CA: Plural Publishing.

Presacco A, Innes-Brown H, Goupell MJ, Anderson S. (2017) Effects of stimulus duration on event-related potentials recorded from cochlear-implant users. Ear Hear 38(6):e389-e393.

Purdy SC, Kelly AS. (2016) Change in speech perception and auditory evoked potentials over time after unilateral cochlear implantation in postlingually deaf adults. In: Seminars in Hearing (Vol. 37, pp. 62-73). Thieme Medical Publishers.

Rakszawski B, Wright R, Cadieux JH, Davidson LS, Brenner C. (2016) The effects of preprocessing strategies for pediatric cochlear implant recipients. J Am Acad Audiol 27(2):85-102.

Shallop JK. (1993) Objective electrophysiological measures from cochlear implant patients. Ear Hear 14(1):58-63.

Svirsky MA, Robbins AM, Kirk KI, Pisoni DB, Miyamoto RT. (2000) Language development in profoundly deaf children with cochlear implants. Psychol Sci 11(2):153-158.

Vermeire K, Brokx JPL, Wuyts FL, Cochet E, Hofkens A, Van de Heyning PH. (2005) Quality-of-life benefit from cochlear implantation in the elderly. Otol Neurotol 26(2):188-195.

Woods DL, Alain C, Covarrubias D, Zaidel O. (1995) Middle latency auditory evoked potentials to tones of different frequency. Hear Res 85(1-2):69-75. 